\title{
Holographic interconnections in photorefractive waveguides
}

\author{
David J. Brady and Demetri Psaltis
}

\begin{abstract}
An architecture for implementing large scale holographic interconnections in photorefractive waveguides is described. Methods for controlling the hologram using unguided light are considered and experimentally demonstrated.
\end{abstract}

\section{Introduction}

Volume holograms in waveguides offer a straightforward means of interfacing dynamically reconfigurable interconnections with integrated optoelectronic devices. Previous uses of thick holograms in waveguides have included grating couplers and distributed feedback lasers. ${ }^{1}$ Holograms for dynamic applications have also been considered, especially in photorefractive crystals. A review of work on photorefractive holograms in waveguides is presented by Wood et $a l^{2}{ }^{2}$ The potential for information storage in integrated volume holograms was considered by Jannson, ${ }^{3}$ who showed that the number of degrees of freedom which can be stored in a planar waveguide hologram scales with the area of the hologram divided by the square of the guided wavelength. In this paper we consider integrated holograms for large scale linear transformations. We rederive Jannson's result in this context and describe a novel method for recording holograms in a waveguide using unguided light. The use of unguided light dramatically simplifies the problem of forming appropriate holograms with high dynamic range. We present experimental results for holograms in photorefractive waveguides formed by titanium indiffusion in $\mathrm{LiNbO}_{3}$.

A thick hologram in a waveguide may be regarded as a vector-matrix multiplier mapping an input vector corresponding to the incident field to an output vector

\footnotetext{
When this work was done both authors were with California Institute of Technology, Department of Electrical Engineering, Pasadena, California 91125; D. J. Brady is now with University of Illinois at Urbana-Champaign, Department of Electrical \& Computer Engineering, Urbana, Illinois 61801.

Received 2 May 1990.

0003-6935/91/172324-10\$05.00/0.

(C) 1991 Optical Society of America.
}

corresponding to the diffracted field via a matrix represented by the interconnecting hologram. In Sec. II we develop this analogy in detail and derive basic relationships for this system. Section III describes methods for controlling a hologram in the waveguide with unguided light. Section IV describes an experimental demonstration of multiple grating holograms written with unguided light and reconstructed with guided light. Section V briefly considers applications for integrated volume holograms.

\section{Integrated Optical Vector-Matrix Multiplier}

An architecture for an integrated optical vectormatrix multiplier is shown in Fig. 1.4 Light from each of $N_{1}$ input channel waveguides is coupled into a slab waveguide and collimated by a waveguide lens. The collimated beams are diffracted by a volume hologram distributed over an area $A$ of the slab waveguide. The diffracted signals are focused by a second integrated lens into a set of $N_{2}$ output channel waveguides. In this section we derive conditions such that a unique holographic grating couples light from each input channel to each output channel. If the gratings are weak enough that the hologram does not deplete the input beams, the fraction of the $l$ th input which is diffracted to the $m$ th output is linear in the corresponding grating amplitude and the diffraction of light from the inputs to the outputs may be regarded as a vector-matrix multiplication.

As shown Fig. 1, we define the $z$-axis to the principal axis of propagation in the waveguide. The $x$-axis is normal to the plane of the waveguide and the $y$-axis is transverse to the $z$-axis in the plane of the waveguide. The origin of the coordinate system is at the entrance face of the hologram at the center of the waveguide. We assume that the waveguide has a boundary with air at $x=-d / 2$ and a substrate boundary at $x=d / 2$. In the absence of a holographic perturbation, the regions $x<d / 2,-d / 2<x<d / 2$, and $d / 2<x$ are assumed to be homogeneous and isotropic. The indices of refrac- 


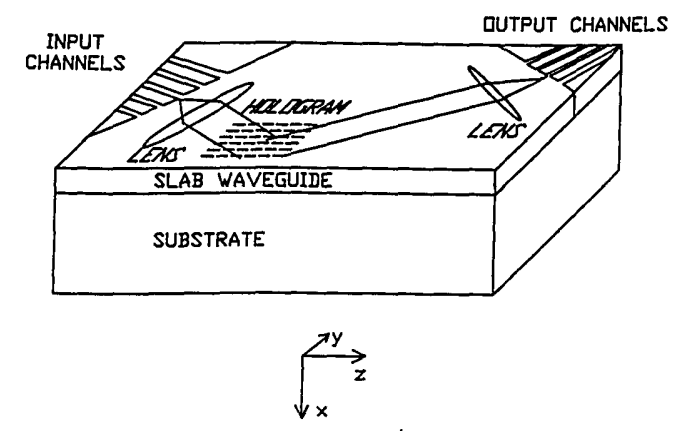

Fig. 1. Architecture for a holographic integrated optical vectormatrix multiplier.

tion of these regions are $n_{1}, n_{2}$, and $n_{2}$, respectively. We assume that $n_{1}<n_{3}<n_{2}$. The guided modes of this system have been analyzed by a number of workers. ${ }^{5,1}$ In considering the system of Fig. 1, the most important results of this analysis are that the dependence of the guided modes on $x$ is separable from the dependence on $z$ and $y$ and that the wave normal curve for the longitudinal components of the guided modes is a circle. We assume that $d$ and $\left(n_{1}, n_{2}, n_{3}\right)$ are such that the slab waveguide in Fig. 1 supports only one mode in $x$.

This system is most easily visualized in the Fourier domain. The signal from the $l$ th input channel is transformed into a plane wave propagating in a unique direction, with wave vector $\mathbf{k}^{(l)}$. Similarly, the signal incident on the $m$ th output channel is associated with the wave vector $\mathbf{k}^{(m)}$. The hologram is a perturbation, $\Delta n(\mathbf{r})$, to the index of refraction of the waveguiding material. We can expand $\Delta n(\mathbf{r})$ in a Fourier series:

$$
\Delta n(\mathbf{r})=\sum_{g} \kappa_{g} \exp \left[j \mathbf{K}_{g} \cdot \mathbf{r}\right),
$$

where $\kappa_{g}$ and $\mathbf{K}_{g}$ are the amplitude and the wave vector of the gth Fourier component. Although only one index is shown, the sum in Eq. (1) is over a set of wave vectors $\mathbf{K}_{g}$ which spans three dimensions. Since the purpose of the hologram is to couple guided modes, we are not interested in Fourier components corresponding to $\mathbf{K}_{g}$ with large components in the out-of-plane $(\hat{x})$ direction. In the region $x<-d / 2, \Delta n(\mathbf{r})=0$.

The components of the wave vectors of the guided modes of a planar waveguide are constrained to lie on a set of wave normal curves in the guiding plane. In a single-mode homogeneous, isotropic waveguide, these curves take the form of a circle of radius $k=2 \pi n_{\text {eff }} / \lambda$, where $\lambda$ is the free-space wavelength of the guided light and $n_{\text {eff }}$ is the effective index of the waveguide. A set of input and output wave vectors on this wave normal circle is sketched in Fig. 2. Each of these wave vectors corresponds to an eigenmode of the waveguide. In the presence of a holographic perturbation, the plane waves corresponding to these wave vectors cease to be eigenmodes. If the perturbation is weak, however, the fields in the waveguide can be described in terms of the unperturbed eigenmodes weighted by slowly varying

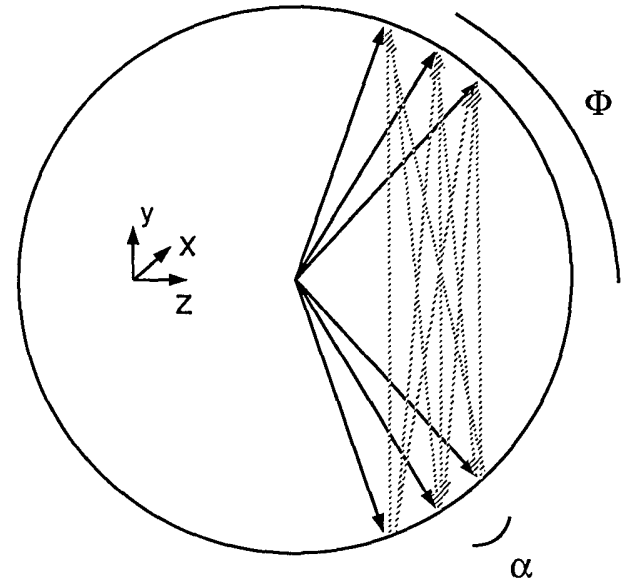

Fig. 2. Optical wave vectors of the coupled guided beams on the wave normal curve in the guided plane. Connecting grating wave vectors are shown as dashed lines.

amplitude functions. These amplitude functions are determined using coupled wave theory. ${ }^{6}$ The coupling strength between the $l$ th input and the $m$ th output is proportional to the amplitude of the Fourier component of $\Delta n$ with wave vector

$$
\mathbf{K}_{l m}=\mathbf{k}^{(m)}-\mathbf{k}^{(l)} \text {. }
$$

In Fig. 2 this condition means that the wave vector of the grating which couples an input to an output must join the end points of the coupled optical wave vectors. A set of grating wave vectors coupling the fields represented in the figure is shown by the dashed lines.

Let $\phi$ be the angle between the $z$-axis and the central plane wave components of the incident and diffracted fields; $\alpha$ is the angle between adjacent plane wave components. The angle between the wave vector corresponding to the $l$ th input channel and the $z$-axis is $-(\phi+l \alpha)$. The wave vector of the $l$ th input is

$$
\begin{aligned}
& \mathbf{k}^{l)}=k \cos (\phi-l \alpha) \hat{z}-k \sin (\phi+l \alpha) \hat{y} \\
& \approx k[\cos (\phi)-l \alpha \sin (\phi)] \hat{z}-k[\sin (\phi)-l \alpha \cos (\phi)] \hat{y},
\end{aligned}
$$

where $\hat{x}, \hat{y}$, and $\hat{z}$ are unit vectors along the corresponding axes and we have made the paraxial approximation, $l \alpha \ll 1$. The angle between the wave vector corresponding to the $m$ th output channel and the $z$ axis is $\phi+m \alpha$. The corresponding wave vector is

$$
\begin{aligned}
\mathbf{k}^{(m)}= & k \cos (\phi+m \alpha) \hat{z}+k \sin \phi+m \alpha) \hat{y} \\
& \approx k[\cos (\phi)-m \alpha \sin (\phi)] \hat{z}+k[\sin (\phi)+m \alpha \cos (\phi)] \hat{y} .
\end{aligned}
$$

Field $\mathbf{E}(\mathbf{r})$ in the waveguide may be represented by a weighted sum of the incident and diffracted fields. We represent the $x$ dependence of the guided modes by the normalized transverse field distribution $\mathscr{E}(x)$. Coupling from the input modes to the output modes results in a $z$ dependence in the amplitudes of the field in each mode. Letting $\Psi_{l}(z)$ and $\Phi_{m}(z)$ represent the amplitudes of the fields corresponding to the $l$ th and $m$ th input and output channels, respectively, the field in the waveguide is 


$$
\begin{aligned}
\mathbf{E}(\mathbf{r})=\mathscr{E}(x) \sum_{l} \Psi_{l}(z) \exp \left[j k^{(l)} \cdot \rho\right] \hat{e}_{l} & \\
& +\varepsilon(x) \sum_{m} \Phi_{m}(z) \exp \left[j k^{(m)} \cdot \rho\right] \hat{e}_{m},
\end{aligned}
$$

where $\rho$ is the position vector in the $y-z$ plane and $\hat{e}_{l}$ and $\hat{e}_{m}$ represent the polarization vectors of the corresponding modes. In the absence of a hologram $\Psi_{l}$ and $\Phi_{m}$ are independent of $z$. The effect of the holographic coupling is determined by substituting $\mathbf{E ( r )}$ into the wave equation:

$$
\nabla \times \nabla \times \mathbf{E}-n^{2}(\mathbf{r}) k^{2} \mathbf{E}=0,
$$

where $k=2 \pi / \lambda$ and $n(\mathbf{r})$ is the index of refraction. Applying the slowly varying envelope approximation and keeping only the first order in $\Delta n$, this approach yields

$$
\begin{aligned}
\sum_{l} j k_{z}^{(l)} \exp \left[j \mathbf{k}^{(l)} \cdot \boldsymbol{\rho}\right] & \frac{\partial \psi_{l}(z)}{\partial z}+\sum_{m} j k_{z}^{(m)} \exp \left[j \mathbf{k}^{(m)} \cdot \boldsymbol{\rho}\right] \frac{\partial \Phi_{m}(z)}{\partial z} \\
= & \sum_{g} n_{o}(x) k^{2} \kappa_{g} \exp \left(j \mathbf{K}_{g} \cdot \mathbf{r}\right) \\
& \times\left\{\sum_{l^{\prime}} \psi_{l^{\prime}}(z) \exp \left[j k^{\left(l^{\prime}\right)} \cdot \boldsymbol{\rho}\right]+\sum_{m^{\prime}} \Phi_{m^{\prime}}(z) \exp \left[j \mathbf{k}^{\left(m^{\prime}\right)} \cdot \rho\right]\right\},
\end{aligned}
$$

where $n_{o}$ is the index distribution in the absence of the perturbation. The next step is to match terms at identical spatial frequencies, which allows us to convert Eq. (6) into a set of coupled linear equations, with each equation describing how one of the modes is coupled to the rest. While the wave vectors of the guided modes are confined to the $y-z$ plane, the grating wave vectors can point in any direction [note the distinction between $\rho$ and $r$ in Eq. (7)]. Since we are interested in couplings between guided modes, however, we may assume that only gratings with wave vectors approximately in the guided plane have been recorded. Integrating Eq. (7) across the waveguide in the $x$-direction, we obtain

$$
\begin{gathered}
\sum_{l} j k_{z}^{(l)} \exp \left[j \mathbf{k}^{(l)} \cdot \rho\right] \frac{\partial \Psi_{l}(z)}{\partial z}+\sum_{m} j k_{z}^{(m)} \exp \left[j \mathbf{k}^{(m)} \cdot \rho\right] \frac{\partial \Psi_{m}(z)}{\partial z} \\
=\sum_{\mathbf{K}_{k^{\prime}}} \frac{\sin \left(k_{g x} d / 2\right)}{K_{g x} d / 2} n_{2} k^{2} \kappa_{g} \exp \left[j \mathbf{K}_{g} \cdot \rho\right) \\
\times\left[\sum_{l^{\prime}} \Psi_{l^{\prime}}(z) \exp \left[j \mathbf{k}^{\left(l^{\prime}\right)} \cdot \rho\right]+\sum_{m^{\prime}} \Phi_{m^{\prime}}(z) \exp \left[j \mathbf{k}^{\left(m^{\prime}\right)} \cdot \rho\right]\right] .
\end{gathered}
$$

The coupling terms due to a given $\mathbf{K}_{g}$ are significant only if $\left|K_{g x}\right|$ is small compared to $4 \pi / d$. We assume that this condition is satisfied for all $\mathbf{K}$ corresponding to nonzero $\kappa_{g}$. In this case, $\sin \left(K_{g x} d / 2\right) / K_{g x} d / 2 \approx 1$. Equation (8) must hold independently for each distinct Fourier component. Multiplying Eq. (8) by $\exp \left[-j \mathbf{k}^{(s)} \cdot \rho\right]$ and integrating over the area of the hologram allow us to replace $\exp \left[-j \mathbf{k}^{(r)} \cdot \rho\right]$ in each term with

$$
\iint \exp \left(j\left[\mathbf{k}^{(r)}-\mathbf{k}^{(s)}\right] \cdot \rho\right\} d \rho=\frac{\sin \left[\Delta k_{r s(y)} L_{y} / 2\right]}{\Delta k_{r s(y)} / 2} \frac{\sin \left[\Delta k_{r s(z)} L_{z} / 2\right]}{\Delta k_{r s(z)} / 2},
$$

where $\Delta \mathbf{k}_{r s}=\mathbf{k}^{(r)}-\mathbf{k}^{(s)}$ and the integral is over the extents of the hologram in $y$ and $z, L_{y}$ and $L_{z}$. The separation between the wave vectors of the input and output modes is chosen such that the integral of Eq. (9) is much less than one if $r \neq s$. This implies that

$$
\alpha>\frac{\lambda}{2 \pi n_{\mathrm{eff}} L_{y} \cos \phi}, \frac{\lambda}{2 \pi n_{\mathrm{eff}} L_{z} \sin \phi} .
$$

In this case, multiplying Eq. (8) by $\exp \left[-j \mathbf{k}_{(m)} \cdot \rho\right]$ and integrating over the guided plane yields

$$
\begin{aligned}
j k_{z}^{(m)} L_{y} L_{z} \frac{\partial \Phi_{m}(z)}{\partial z}=\sum_{g} \sum_{l} \frac{\sin \left[\Delta K_{g l m(y)} L_{y} / 2\right]}{\Delta K_{g l m(y)} / 2} \\
\quad \times \frac{\sin \left[\Delta K_{g l m(z)} L_{z} / 2\right]}{\Delta K_{g l m(z)} / 2} n_{2} k^{2} \kappa_{g} \Psi_{l}(z),
\end{aligned}
$$

where $\Delta \mathbf{K}_{g l m}=\mathbf{K}_{g}-\left[\mathbf{k}^{(l)}-\mathbf{k}^{(m)}\right]$. We have not included couplings between output modes under the assumption that $\Delta K_{g l m} \cdot \mathbf{L} \gg 1$ for all nonzero $\kappa_{g}$ if $l$ and $m$ are both output modes. The coupling term between the $m$ th output mode and $l$ th input mode is strong only if $\sin \left[\Delta K_{g l m(y)} L_{y} / 2\right] /\left[\Delta K_{g l m(y)} / 2\right]$ and $\sin \left[\Delta K_{g l m(z)} L_{z} / 2\right] /$ $\left[\Delta K_{g l m(z)} / 2\right]$ are nonvanishing. This is the case if

$$
\begin{aligned}
& \left|\Delta \mathbf{K}_{g l m(y)}\right|<\frac{\pi}{L_{y}}, \\
& \left|\Delta \mathbf{K}_{g l m(z)}\right|<\frac{\pi}{L_{z}} .
\end{aligned}
$$

When $\alpha$ satisfies Eq. (10), Eqs. (12) and (13) are satisfied only if $\mathbf{K}_{g}=\mathbf{K}_{l m} \equiv \mathbf{k}^{(l)}-\mathbf{k}^{(m)}$. Discarding terms from Eq. (11) for which these conditions do not hold yields

$$
j k_{z}^{(m)} \frac{\partial \Phi_{m}(z)}{\partial z}=\sum_{l} n_{2} k^{2} \kappa_{l m} \Psi_{l}(z),
$$

where $\kappa_{l m}$ is the amplitude of the grating at spatial frequency $\mathbf{K}_{l m}$. A similar equation may be derived to describe coupling from the output modes to the input modes.

Since we are interested in using the system of Fig. 1 to implement linear transformations, we assume that the diffraction efficiency of the hologram is weak. In this case, the input signals may be assumed to be undepleted by the hologram. Let $H_{l m}=-j L_{z} n_{2} k^{2} \kappa_{l m} /$ $k_{z}^{(m)}$. The solution to Eq. (14) under the assumption of undepleted inputs is

$$
\Phi=\overline{\bar{H}} \Psi \text {, }
$$

where $[\boldsymbol{\Phi}]_{m}=\Phi_{m}\left(l_{z}\right),[\boldsymbol{\Psi}]_{l}=\Psi_{l}=\Psi_{l}(0)$, and we assume that $\Phi_{m}(0)=0$. Since each component of $\bar{H}$ corresponds to an independent grating, appropriately selecting the grating amplitudes allows us to use the hologram to implement an arbitrary linear transformation.

Assuming a diffraction-limited system, the spatial bandwidth of the input and output fields is $B=2 \pi L_{y}$ $\cos \phi / \lambda f$, where $f$ is the focal length of the waveguide lenses. The number of modes which may be used in the input and output fields, $R$, is equal to $B$ divided by the separation in Fourier space between modes 
$2 \pi n_{\text {eff }} \alpha / \lambda$. Substituting for $\alpha$ from Eq. (10) and assuming that $L_{z} \sin \phi=L_{y} \cos \phi, \quad R=A \sin \phi \cos \phi / \lambda f$, where $A=L_{z} L_{y}$. The number of connections a hologram in the plane can make between channels is

$$
R^{2}=\frac{A^{2} \sin 2 \phi}{2 \lambda^{2} f^{2}}
$$

If $f^{2}$ is of the same order as $A, R^{2}$ scales as $A / \lambda^{2} .^{3}$ Assuming $L_{y} \cos \theta / f=1, \quad A=1 \mathrm{~cm}^{2}, \lambda=0.5 \mu \mathrm{m}$, and $\phi$ $=\pi / 6, \quad R^{2}=10^{8}$.

The possibility of coupling between guided and unguided modes is not considered in Eq. (7) because such coupling is not expected to occur for this system. The minimum mismatch between the $\hat{x}$ components of the zeroth-order TE mode and a radiation mode is

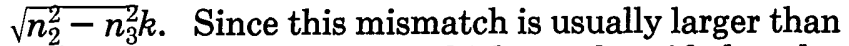
$4 \pi / d$, grating wave vectors which couple guided modes will not usually also couple guided modes with radiation modes. Note that volume holograms in waveguides differ from grating couplers. Conventional grating couplers usually involve a relatively large perturbation, for example, a relief grating, and are much thinner than the waveguide so that the Bragg mismatch normal to the waveguide is not a problem.

\section{Formation of Integrated Optical Volume Holograms}

In this section we consider methods for recording a hologram in a slab waveguide using upguided light incident from above the waveguide (along the $x$-axis in Fig. 1). Recording with unguided light yields two advantages over recording with guided light. First, unguided recording avoids unintended perturbations to the waveguide. If the waveguide is sensitive to the guided beams it is difficult to avoid crosstalk between input beams and between output beams and damage to the waveguide outside of the holographic region. With unguided control, the unintended gratings which give rise to crosstalk are not recorded. The problem of damage to the waveguide can be overcome if the waveguide is not sensitive to the guided beams. In a photorefractive material a threshold wavelength exists beyond which holograms may no longer be written. This allows us to use a guided wavelength that does not damage the waveguide while using a shorter unguided wavelength to record the hologram. The second advantage of unguided recording is that it allows us to write the hologram with a single exposure. Multiple exposures are needed to form an arbitrary hologram with guided recording beams. Recording with multiple exposures is in general undesirable because it greatly complicates dynamic control of the hologram and because it is difficult to maintain a large dynamic range in multiply exposed holograms.

We consider two algorithms for recording a hologram in the guided plane. In the first case each of the $N_{1} N_{2}$ gratings in the plane is formed using a distinct pair of control beams. In the second case the gratings are formed using a single reference beam and $N_{1} N_{2}$ beams generated by a spatial light modulator (SLM). The advantage of the first approach is that all the gratings may be recorded in the plane with no out-of-

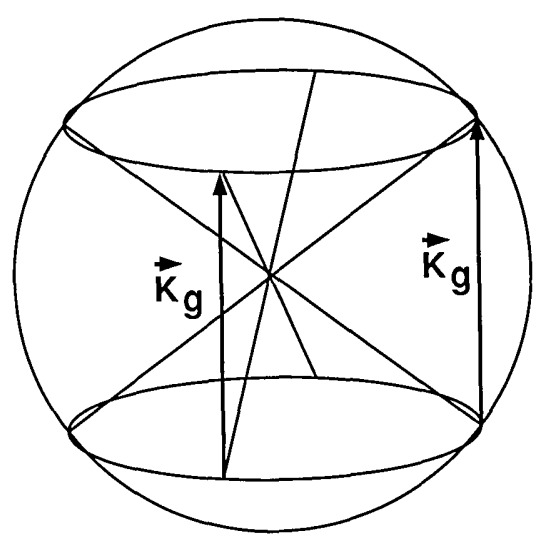

Fig. 3. Wave normal surface showing a grating wave vector and its associated degeneracy cone.

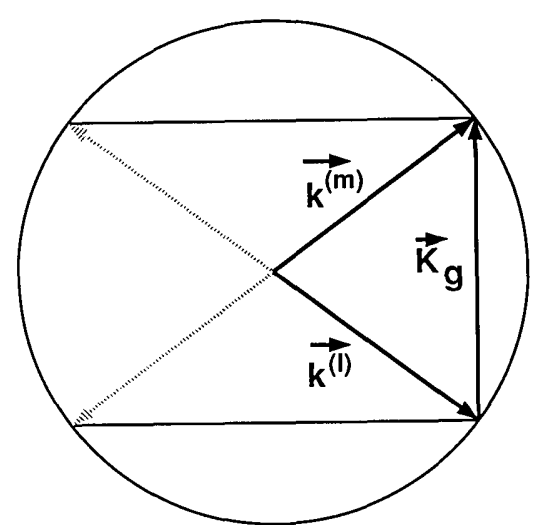

Fig. 4. Projection of the wave normal surface onto the guided plane.

plane components. The second approach sacrifices precision in the spatial orientation of the recorded gratings to simplify the generation of the recording beams and improve the modulation depth with which each grating is recorded.

The distinction between the two recording techniques can be clarified using a simple graphic technique. At most one pair of guided beams, and their conjugates, can satisfy the Bragg condition, Eq. (2), for a grating with wave vector $\mathbf{K}_{g}$. The Bragg condition may, however, be satisfied for a given $\mathbf{K}_{g}$ by many pairs of unguided optical wave vectors. Consider the 3-D wave normal surface sketched in Fig. 3. Although the wave normal surface for a waveguiding system consists of a curve in the guided plane for each guided mode and a potentially anisotropic pair of surfaces for the radiation modes, we can qualitatively demonstrate coupling behavior with greater simplicity by assuming that the wave normal surface is a sphere. Any pair of points on the surface which are joined by $\mathbf{K}_{\mathrm{g}}$ is Bragg matched to the grating. As sketched in Fig. 3, the locus of such pairs is a cone whose axis is parallel to the grating wave vector. The cone intersects the normal surface in two parallel circles separated from each other by the grating wave vector. A 2-D version of Fig. 3 is formed by projecting the wave normal surface onto the guided plane as sketched in Fig. 4. The circle in this figure is 

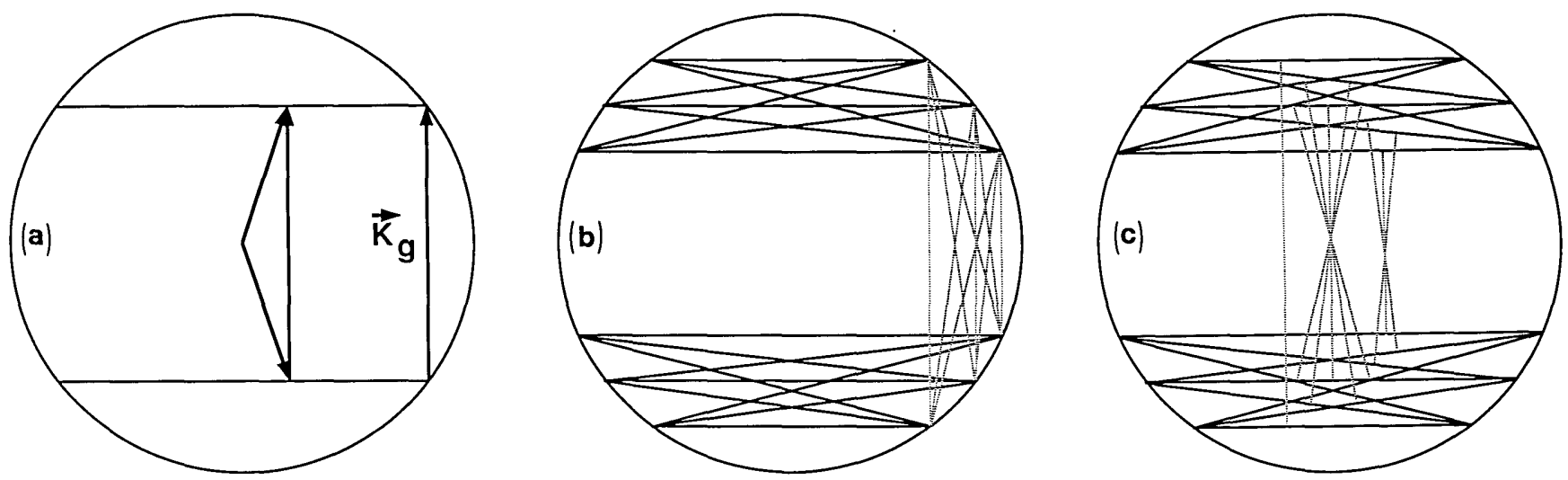

Fig. 5. (a) Projection in the guided plane of the normal surface and a pair of unguided wave vectors. The grating wave vector and its degeneracy lines are also shown. A set of grating wave vectors in the guided plane and their associated degeneracy lines. The set shown interconnects three guided input beams with three guided output beams. (c) Grating wave vectors of (b) displaced along the degeneracy lines.

the projection of the wave normal sphere in the guided plane. The two parallel lines are the projections of the circles on the cone of Fig. 3. We refer to these lines as the degeneracy lines of $\mathbf{K}_{g}$. The separation between the degeneracy lines is $\mathbf{K}_{g}$. The optical wave vectors in the plane which are coupled by the grating lie on the wave normal circle at the points where it intersects the degeneracy lines. The backward propagating conjugates of the coupled pair, shown in the figure as dashed lines, are also coupled. Each point on the interior of the wave normal circle in Fig. 4 is the projection onto the guided plane of a point on the hemisphere of the normal surface below the guided plane. Thus, each point on the interior of the wave normal curve corresponds to an unguided beam incident from above the plane. Points on the degeneracy lines in Fig. 4 are the projections of points on the degeneracy cone. A pair of points separated by $\mathbf{K}_{g}$ on opposite degeneracy lines corresponds to a pair of Bragg matched modes. Although the normal surface need not be a sphere, the wave normal curve may consist of multiple ellipses unconnected to the unguided normal surface, and the wavelengths of the guided and unguided beams may be different, projections of the wave normal surfaces, degeneracy curves, and wave vectors may be constructed to account for these details in a straightforward manner.

Using the first recording method mentioned above, we record $\mathbf{K}_{g}$ using a pair of wave vectors separated by $\mathbf{K}_{g}$ on opposite degeneracy lines. As an example, Fig. 5 (a) shows the projection in the guided plane of a pair of unguided optical wave vectors which could be used to record the grating of Fig. 4. Of course, our goal is to simultaneously record many gratings in the plane. Figure 5(b) shows a set of grating wave vectors (as dotted lines) and the degeneracy lines associated with them. For simplicity, the optical wave vectors coupled by these gratings are not shown. To record these gratings from above the plane, we must select an appropriate pair of optical signals for each pair of degeneracy lines. Crosstalk between the recording signals need not concern us because gratings between optical wave vectors which are not on opposite degeneracy lines will in general be able to couple signals in the plane. In Fig. 5(c) we have translated the grating wave vectors of Fig 5(b) along the associated degeneracy lines. Each end point of a grating wave vector corresponds to an unguided optical wave vector which is to be used to control that grating. The recording process consists of simultaneously exposing the waveguide to the beams corresponding to the end points of the grating wave vectors. The amplitude of each recorded grating is proportional to the amplitudes of the corresponding pair of recording beams relative to the beam recording amplitude.

This recording method is unattractive for two reasons. First, since each grating is written by a separate pair of control beams, $2 N_{1} N_{2}$ spatially disperse signals must be controlled independently. Second, the modulation depth of the fringe pattern corresponding to each grating is weak when all $N_{1} N_{2}$ gratings are recorded with separate pairs of control beams. A much stronger modulation depth is achieved if all $\mathrm{N}_{2} \mathrm{~N}_{2}$ gratings are written using a single reference beam. ${ }^{4}$ However, a grating can be written from above the plane only when both of the writing beams lie on its degeneracy lines. If all the gratings are to be recorded with a single reference beam, that beam must lie on one of the degeneracy lines of each grating. A set of grating wave vectors may be recorded with a single reference beam only if all the corresponding degeneracy lines intersect in a single point. Since pairs of degeneracy lines corresponding to different grating wave vectors intersect in at most one pair of points, such an intersection point is impossible if there is fan-in or fan-out in the interconnection matrix. The second recording method we consider overcomes this dilemma by accepting a tilt out of the guided plane in the recording gratings. Such a tilt is possible because the readout beams are confined to a thin guiding region. As seen in Eq. (8), the component of the grating wave vector out of the guiding plane may be as large as $\pi / d$ before the coupling strength of an otherwise Bragg matched grating is seriously affected. Since the waveguide may be only a few wavelengths 


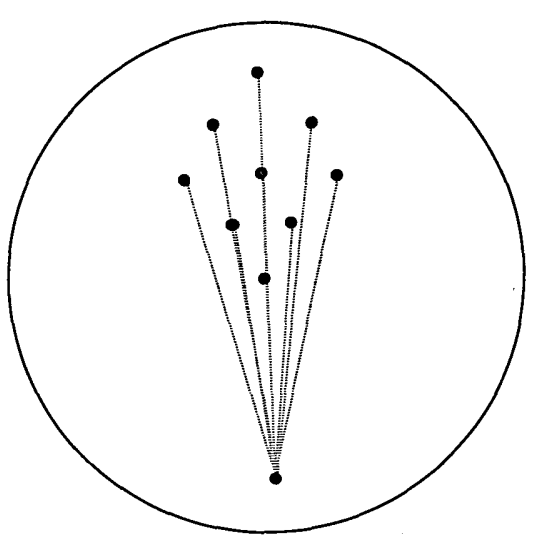

Fig. 6. Projection in the guided plane of the end points of a set of unguided recording beams. The lines in the figure correspond to the grating wave vectors of Fig. 5. The grating wave vectors have been shifted off their degeneracy lines to allow recording with a single reference.

thick, guided beams may reconstruct gratings with fairly sizable out-of-plane components.

If we are not concerned about Bragg mismatch out of the plane of the waveguide, we can record the hologram with any group of optical signals which record gratings with planar components matched to the desired gratings. The analog of Fig. 5(c) for this case is shown in Fig. 6, which contains a line between a pair of end points for each of the grating wave vectors of Fig. 5(b). An end point of each of the grating wave vectors lies on a single reference point. Since the grating wave vectors no longer join points on their degeneracy lines, the actual gratings recorded between the reference beam and the signal beams have out-of-plane components. However, if the out-of-plane component of the actual grating wave vector recorded for each desired grating wave vector is less than $\pi / d$, the gratings recorded using the beams suggested by Fig. 6 will implement the interconnection pattern shown in Fig. 5(b). Note that, while any unguided reference beam could be used in recording the hologram, the magnitude of the outof-plane components of the recorded gratings depends on the reference chosen. In the remainder of this section we describe a specific recording architecture in which the constraint on the out-of-plane components of the recorded gratings can be satisfied for large interconnection matrices.

An architecture for recording a hologram in the waveguide using a single reference is shown in Fig. 7. The hologram is formed between the Fourier transform of the signal recorded on a spatial light modulator and a plane wave reference. The SLM consists of a 2$\mathrm{D}$ array of $S$ independently controllable pixels. Each pixel controls a single grating in the waveguiding plane. We refer to the pixel that controls the $(l m)$ th grating as the $(l m)$ th pixel. The SLM lies in the $y^{\prime}-z^{\prime}$ plane. The optical axis of the SLM-Fourier lens system is along $x^{\prime}$. The optical axis for light propagating in the waveguide is along $z ; x$ is normal to the waveguide; and $y$ is transverse to the propagation direction

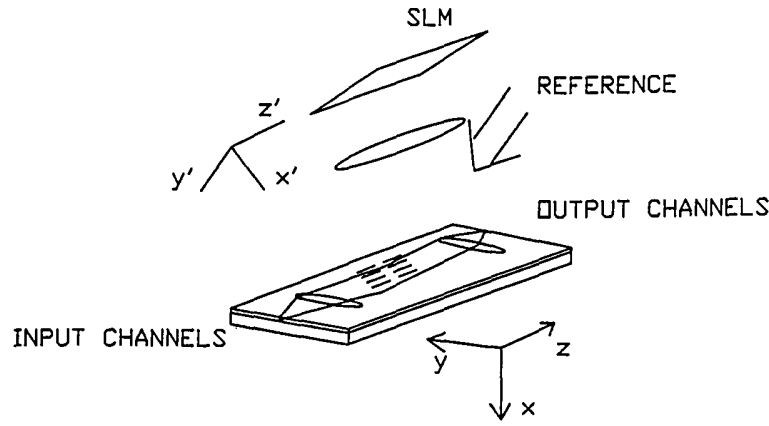

Fig. 7. Architecture for controlling gratings in the guided plane using a single reference and an SLM.

in the plane of the waveguide. The $x^{\prime} y^{\prime} z^{\prime}$ coordinate system corresponds to the $x y z$ system rotated by an angle $-\theta$ about $z$. The reference beam is assumed to propagate in a direction normal to $z$ and at an angle $\theta$ with respect to $x$. The geometry in the plane is the same as in Fig. 1.

The cross-sectional area at the plane of the waveguide of a beam of light collimated from one pixel of the SLM is $\lambda_{r}^{2} F^{2} / \delta$, where $\delta$ is the area of a single pixel, $\lambda_{r}$ is the wavelength of the recording light, and $F$ is the focal length of the Fourier lens. If

$$
\lambda_{r}^{2} F^{2} / \delta>\mathrm{A}
$$

where $A$ is the area of the holographic interaction region in the waveguide, the light generated by a pixel centered at $\left(y^{\prime}, z^{\prime}\right)$ is to a good approximation a plane wave propagating with the wave vector:

$$
\begin{aligned}
& \mathbf{k}=k_{r}\left[1-\frac{\left(u^{2}+v^{2}\right)}{2 k_{r}^{2}}\right] \hat{x}^{\prime}+u \hat{y}^{\prime}+v \hat{z}^{\prime} \\
&=\left\{k_{r}\left[1-\frac{\left(u^{2}+v^{2}\right)}{2 k_{r}^{2}}\right] \cos \theta+u \sin \theta\right\} \hat{x} \\
&+\left\{u \cos \theta-k_{r}\left[1-\frac{\left(u^{2}+v^{2}\right)}{2 k_{r}^{2}}\right] \sin \theta\right\} \hat{y}+v \hat{z},
\end{aligned}
$$

where $u=k_{r} y^{\prime} / F, \quad v=k_{r} z^{\prime} / F$, and $k_{r}=2 \pi / \lambda_{r}$. Let $\mathbf{k}_{m}$ be the wave vector for light collimated from the $(l m)$ th pixel; $u_{l m}$ and $v_{l m}$ correspond to $u$ and $v$ for the $(l m)$ th pixel. Let $H_{l m}$ represent the optical field at the $l m$ th pixel. The field due to the SLM near the plane of the waveguide may be written as

$$
\begin{aligned}
U(x, y, z)= & \exp \left[j k_{r}(x \cos \theta-y \sin \theta)\right] \\
\times \sum_{l m} H_{l m} \exp [ & \left.-j k_{r}(x \cos \theta-y \sin \theta) \frac{\left(u_{l m}^{2}+v_{l m}^{2}\right)}{2 k_{r}^{2}}\right] \\
& \times \exp \left\{j\left[u_{l m}(y \cos \theta+x \sin \theta)+v_{l m} z\right]\right\} .
\end{aligned}
$$

The reference field is $R=\exp \left[j k_{r}(x \cos \theta+y \sin \theta)\right]$. The interference pattern between the signal and reference fields is 


$$
\begin{aligned}
I(x, y, z)=\exp \left(-j 2 k_{r} y \sin \theta\right) \sum_{l m} H_{l m} \\
\times \exp \left[-j k_{r}(x \cos \theta-y \sin \theta) \frac{\left(u_{l m}^{2}+v_{l m}^{2}\right)}{2 k_{r}^{2}}\right] \\
\quad \times \exp \left\{j\left[u_{l m}(y \cos \theta+x \sin \theta)+v_{l m} z\right]\right\}+\text { c.c }
\end{aligned}
$$

Assuming that the holographic perturbation is linear in $I(x, y, z)$, the amplitude of the Fourier component of the perturbation at spatial frequency

$$
\begin{aligned}
\mathbf{K}_{l m}= & {\left[u_{l m} \sin \theta-\cos \theta \frac{\left.u_{l m}^{2}+u_{l m}^{2}\right)}{2 k_{r}}\right] \hat{x} } \\
& -\left[2 k_{r} \sin \theta-u_{l m} \cos \theta-\sin \theta \frac{\left(u_{l m}^{2}+v_{l m}^{2}\right)}{2 k_{r}}\right] \hat{y}+v_{l m} \hat{z}
\end{aligned}
$$

is the proportional to $H_{l m}$.

Suppose that we wish to control the interconnection pattern of Fig. 2 using the architecture of Fig. 7. To control the $l m$ th grating with the $l m$ th pixel we must select $u_{l m}, u_{l m}$, and $\theta$ such that the components of $\mathbf{K}_{l m}$ in the guided plane given by Eq. (21) are equal to $\mathbf{K}_{l m}$. By assuming that $\left(u_{o o}, v_{o o}\right)=(0,0)$ and discarding second-order terms, we find $k_{r} \sin \theta=k \sin \phi$ and

$$
\begin{aligned}
& u_{l m}=(l+m) \alpha k \frac{\cos \phi}{\cos \theta}, \\
& u_{l m}=(l-m) \alpha k \sin \phi ;
\end{aligned}
$$

$\mathbf{K}_{l m}$ and $\phi$ are the same as in the previous section. Comparing Eq. (22) with Eq. (18) we see that the lmth grating controlled by the field on a pixel centered at $\left(y_{l m^{\prime}}, z_{l m}{ }^{\prime}\right)=[l+m) \alpha F\left(\lambda_{r} \cos \phi / \lambda \cos \theta\right),(l-m) \alpha F\left(\lambda_{r} / \lambda\right)$ $\sin \phi]$. Gratings which couple the $l$ th input to the output channels are controlled by the pixels on the line:

$$
y^{\prime}=-\frac{z^{\prime}}{\tan \phi \cos \theta}+2 l \alpha F \frac{\lambda_{r} \cos \phi}{\lambda \cos \theta} .
$$

Gratings which couple light from input channels to the $m$ th output are controlled by pixels on the line:

$$
y^{\prime}=\frac{z^{\prime}}{\tan \phi \cos \theta}+2 m \alpha F \frac{\lambda_{r} \cos \phi}{\lambda \cos \theta} \text {. }
$$

To ensure that the single reference exposure method does not violate the Bragg condition out of the plane, we require that the $x$-component of Eq. (21) be less than $\pi / d$ :

$$
\left|\left[u_{l m} \sin \theta-\cos \theta \frac{\left(u_{l m}^{2}+v_{l m}^{2}\right)}{2 k_{r}}\right]\right|<\frac{\pi}{d} .
$$

Let $r$ be the radius of the active area of the SLM. Equation (25) is satisfied for all $l$ and $m$ if

$$
\left|\left(\frac{k_{r} r}{F} \sin \theta+\cos \theta \frac{k_{r} r^{2}}{2 F^{2}}\right)\right|<\frac{\pi}{d} .
$$

Assuming that $\tan ^{2} \ll\left(k_{r} d\right)$, Eq. (26) simplifies to

$$
r<\sqrt{2 \pi} \frac{F}{\sqrt{k_{r} d}} \text {. }
$$

The number of gratings in the guided plane that we can control using the SLM is $S=\pi r^{2} / \delta$. Substituting from Eq. (27) we find

$$
S<\frac{\lambda_{r} F^{2}}{d \delta} .
$$

Substituting from Eq. (17), we see that Eq. (28) is satisfied if

$$
S=\frac{A}{\lambda_{r} d}
$$

$S$ differs from the number of gratings we can distinguish in the plane, given as $R^{2}$ in Eq. (16), only by the factor $\lambda^{2} / \lambda_{r} d$.

\section{Experimental Results}

In our experiments the SLM and lens of Fig. 7 are simulated by a square-wave grating. The grating lies in the $y^{\prime}-z^{\prime}$ plane. The transmittance of the grating is a $1-\mathrm{D}$ function, $t(\xi)$, which may be described by a Fourier series over harmonics of a fundamental frequency $K_{f} \hat{\xi}$, where $\hat{\xi}$ is a unit vector in the $y^{\prime}-z^{\prime}$ plane. Assuming that a plane wave propagating along the positive $x^{\prime}$ axis is incident on the grating, the wave vector of the $n$th harmonic of the optical field diffracted from the grating is

$$
p^{(n)}=k_{r} \sqrt{1-\frac{n^{2} k_{f}^{2}}{k_{r}^{2}}} x^{\prime}+n k_{f} \hat{\xi} .
$$

Comparing Eq. (30) with Eq. (18), we define $u_{n}=$ $n K_{f} \hat{\xi} \cdot y^{\prime}$ and $v_{n}=n K_{f} \hat{\xi} \cdot z^{\prime}$. The field transmitted by the grating is

$$
U(x, y, z)=\sum_{n} H_{n} \exp \left[j p^{(n)} \cdot \mathbf{r}\right] .
$$

A hologram is formed in the waveguiding plane between this field and the reference beam $R=\exp \left[j k_{r}(x\right.$ $\cos \theta+y \sin \theta)]$. The amplitude of the Fourier component of this hologram at spatial frequency $\mathbf{K}_{n}=p^{(\mathbf{n})}-$ $k_{r}(\cos \theta \hat{x}+\sin \theta \hat{y})$ is proportional to $H_{n}$.

The component of the hologram at spatial frequency $\mathbf{K}_{n}$ couples the $l$ th input to the $m$ th output only if $\mathbf{K}_{n}=$ $\overrightarrow{\mathrm{K}}_{\mathrm{lm}}$. Substituting $u_{n}$ and $v_{n}$ from Eq. (27) into Eq. (19) and solving for $l$ and $m$ we find $\mathbf{K}^{(n)}=\mathbf{K}_{l m}$ when

$$
\begin{aligned}
& l=\frac{n K_{f}}{\alpha k}\left(\cos \gamma \frac{\cos \theta}{\cos \phi}+\frac{\sin \gamma}{\sin \phi}\right), \\
& m=\frac{n K_{f}}{\alpha k}\left(\cos \gamma \frac{\cos \theta}{\cos \phi}-\frac{\sin \gamma}{\sin \phi}\right),
\end{aligned}
$$

where $\gamma=\cos ^{-1}\left(\hat{\xi} \cdot \hat{y}^{\prime}\right)$. If $\tan \gamma=-\cos \theta \tan \phi$, then $l=$ 0 and the $n$th harmonic controls the coupling from the Oth input channel to the $m$ th output channel, where $m$ is given by Eq. (32); $\hat{\xi}$ is parallel to the line of Eq. (23) in this case. Similarly, if $\tan \lambda=\cos \theta \tan \phi, m=0$ and the $n$th harmonic controls the coupling from the output channel corresponding to $m=0$ to the $l$ th input channel.

Figure 8 is a sketch of the grating wave vectors formed in the guided plane by the five lowest-order 


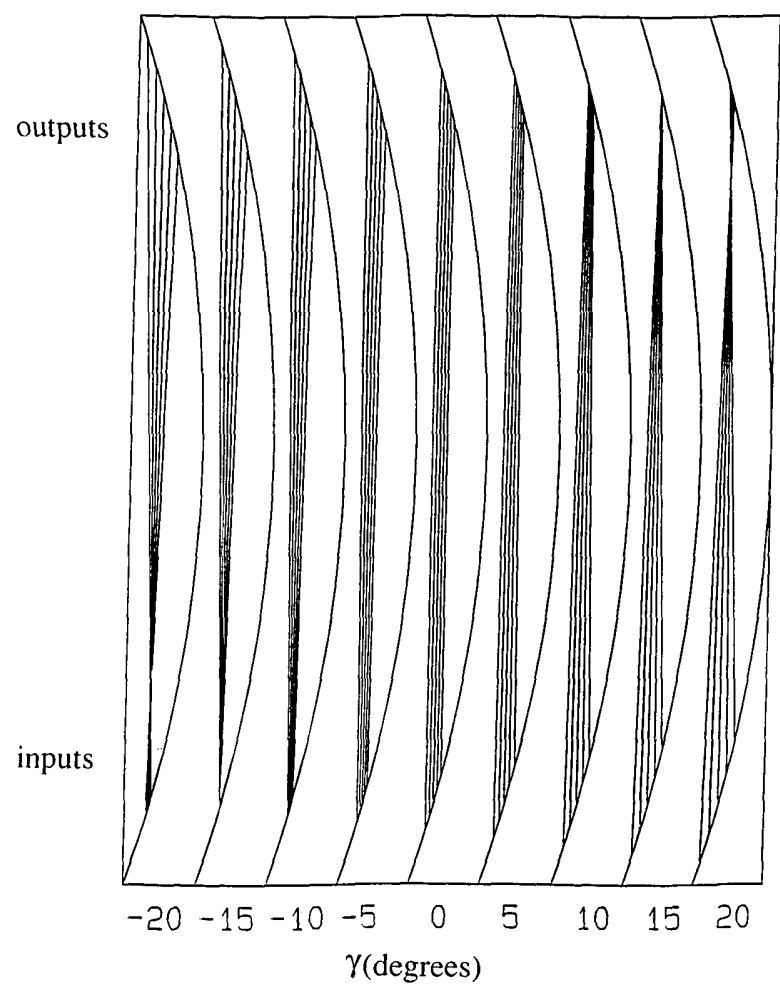

Fig. 8. Grating wave vectors recorded in the guided plane as a function of $\gamma$, the angle of rotation of the control grating. The gratings recorded in the plane due to the zeroth, first, and second orders of the control grating are shown.

Fourier components of a recording grating at various rotations $\gamma$ of the grating in the $y^{\prime}-z^{\prime}$ plane. A section of the wave normal curve and the positions of the five grating wave vectors recorded on the normal surface are shown for each value of $\gamma$. In the case shown $\phi=$ $15^{\circ}, \quad k_{r} / k=1.5$, and $K_{f} / k=0.02$. At $\lambda=-\tan ^{-1}(\cos \theta$ $\tan \phi)=-14.8^{\circ}$, a single input is coupled to five different outputs.

We have recorded photorefractive holograms in single-mode titanium indiffused waveguides on nominally pure $y$-cut $\mathrm{LiNbO}_{3}$ substrates. The entire surface of the substrate was waveguiding and no integrated lenses or channels were constructed for these experiments. Holograms were recorded from above the waveguide using light at $514 \mathrm{~nm}$. The guided TE mode at $633 \mathrm{~nm}$ was coupled into the waveguide using a rutile prism and then used to reconstruct the holograms. The use of undoped $\mathrm{LiNbO}_{3}$ meant that the photorefractive response of the sample was relatively weak. An exposure of $\sim 50 \mathrm{~J} / \mathrm{cm}^{2}$ was used to record holograms. Even at this exposure diffraction of the recording 514-nm beams was weak. Diffraction efficiencies for the guided beams were as high as $5 \%$ in spite of this fact because the length of the waveguide over which these holograms acted was $\approx 1 \mathrm{~cm}$. The thickness of the substrate through which the recording beams passed was $2 \mathrm{~mm}$.

To demonstrate the connection patterns shown in Fig. 8, holograms were formed between a plane wave reference and light diffracted from a Ronchi grating

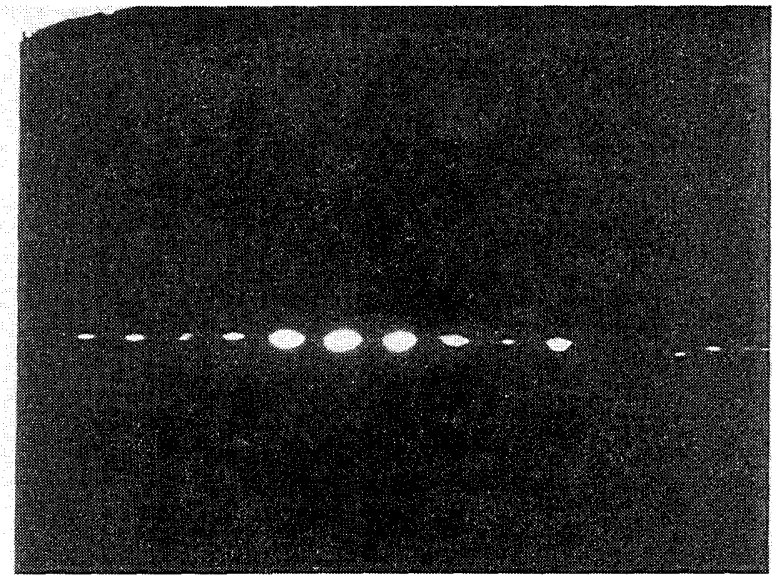

(a)

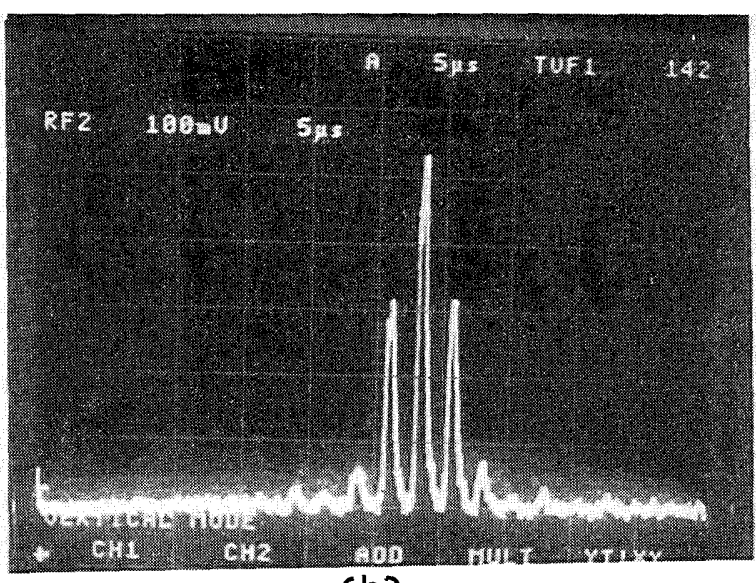

(b)

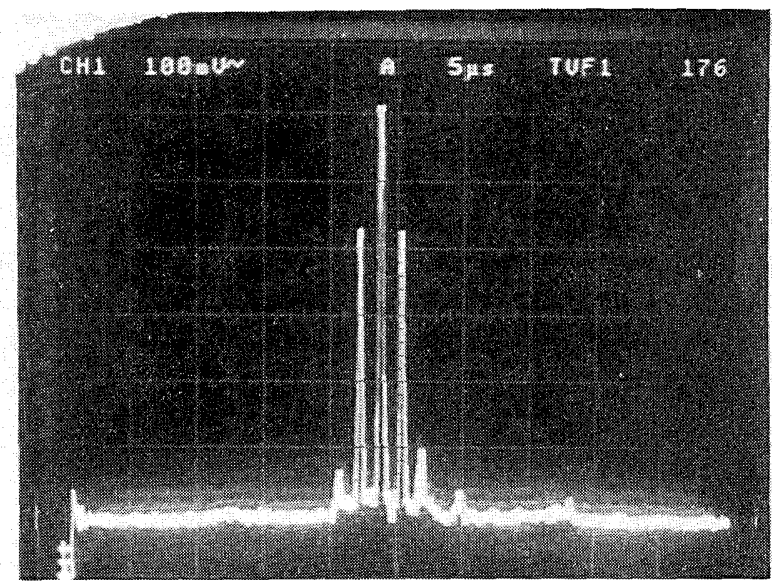

(c)

Fig. 9. Diffracted He-Ne signals at the output of a TI:LiNbO waveguide. The diffracting hologram was recorded using unguided $\mathrm{Ar}^{+}$control beams consisting of a single reference and the diffracted orders of a Ronchi ruling.

with 100 lines/in. By rotating the grating in the $y^{\prime}-z^{\prime}$ plane, we were able to observe variations in the pattern of grating wave vectors stored in the plane similar to those shown in Fig. 8. For most orientations of the Ronchi grating, we found several different angles of incidence for which the guided mode would be diffract- 

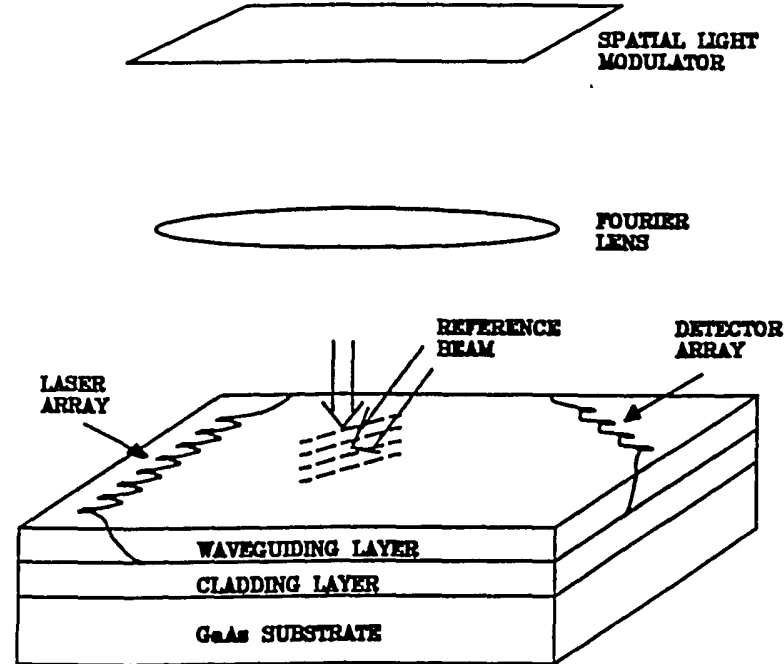

Fig. 10. Architecture for monolithic integration of active devices and photorefractive waveguides in GaAs.

ed into one or more orders. For $\phi=1.5^{\circ}, \theta=1^{\circ}$, and $\gamma$ $=-1.5^{\circ}$, however, only one angle of incidence existed for which the guided mode was diffracted. The field incident at this angle was diffracted into orders corresponding to the diffraction orders of the Ronchi grating. The diffraction orders in the waveguide were end-coupled out and focused on a CCD camera. A photograph of the focused diffraction orders is shown in Fig. 9(a). The fourteen lowest orders are visible. (The angles inside the waveguide are given. The angle between the $\mathrm{Ar}^{+}$beams incident on the substrate was $5^{\circ}$. Small angles were used because the sample we used is not guiding along $y$.)

We have assumed that the amplitude of the hologram we write in the waveguide is proportional to the recording intensity. This is not quite the case for photorefractive holograms that are linear in the modulation depth of the recording fringe pattern. If the intensity is fairly uniform, however, the distinction need not concern us here. Under the assumption of linear recording, the strength of a connection in the plane is proportional to $H_{l m}$. This is confirmed in Figs. 9(b) and (c). Figure 9(b) is a cross section of the optical Fourier transform of the Ronchi grating used to make the hologram of Fig. 9(a). This figure was formed by focusing light passing through the Ronchi grating onto a CCD camera. Figure 9(c) shows, on a slightly different horizontal scale, a cross section of the video image corresponding to Fig. 9(a). This figure shows the relative intensities of the diffracted orders coupled out of the end of the waveguide. Because the transformation from the relative amplitudes of the recording signals generated by the Ronchi grating to the relative amplitudes of the gratings seen by the guided mode is linear for this geometry, the relative amplitudes of the frequency components of Fig. 9(b) are preserved in Fig. 9(c). The noise in the base lines of Fig. 9 is the dark noise of a CCD camera.

While a number of issues, most particularly the problem of integrating collimating and focusing lens arrays with channel arrays, need to be addressed before the vector-matrix multiplier proposed in this paper is demonstrated, the experiment described in this section shows tha the most novel aspect of this system, independent unguided control of multiple grating transformations between guided signals, is practical.

\section{v. Conclusion}

Volume holograms in waveguides are applicable to systems that require fixed or adaptive linear transformations. While the out-of-plane control method described here provides a method for producing fixed integrated holograms, the fact that this method can be used to dynamically control connections in a plane may be more significant. Such a dynamically controlled vector-matrix multiplier would be useful in switching networks or in adaptive artificial neural networks.

Semiconducting photorefractive materials are especially attractive for dynamic implementations of integrated volume holography. This is because photorefractive response times in these materials are relatively fast and, at least in the case of GaAs, integrated technologies are well developed. Using $\mathrm{GaAs}$, we may expect to be able to monolithically construct architectures such as that shown in Fig. 10. In this system an array of laser diodes is dynamically connected to an array of photodetectors using a photorefractive hologram with out-of-plane control. Since photorefractive effects arise on a fairly short time scale in $\mathrm{GaAs},{ }^{7}$ one could expect to reconfigure the interconnection matrix in this device in a few microseconds. Depending on the number of input channels which could be integrated, from $10^{4}$ to $10^{8}$ weighted interconnections could be stored in this device. One problem with integrating a large number of input channels might be the integration of more than 100 lasers on a single chip. This problem could be overcome by fanning out the laser outputs to feed several input channels, each channel being controlled by a simple modulator. The principal difficulty arising in the fabrication of this device is the well-known problem of monolithically integrating active and linear waveguiding regions. While considerable further work is needed to overcome this difficulty, many grating integrated holograms offer interesting new possibilities for holographic information processing.

This work is supported by the Air Force Office of Scientific Research and DARPA.

\section{References}

1. See, for example, R. G. Hunsperger, Integrated Optics: Theory and Technology (Springer-Verlag, Berlin, 1985).

2. V. E. Wood, P. J. Cressman, R. L. Holman, and C. M. Verber, "Photorefractive Effects in Waveguides," in Photorefractive Materials and Their Applications II, P. Gunter and J.-P. Huignard, Eds. (Springer-Verlag, New York, 1989). 
3. T. Jannson, "Information Capacity of Bragg Holograms in Planar Optics," J. Opt. Soc. Am. 71, 342-347 (1981).

4. D. Psaltis and D. J. Brady, "A Photorefractive Integrated Optical Vector-matrix Multiplier," Proc. Soc. Photo-Opt. Instrum. Eng. 835, 153-161 (1987).

5. See, for example, D. Marcuse, Theory of Dielectric Optical Waveguides (Academic, New York, 1979).
6. H. Kogelnik, "Coupled Wave Theory for Thick Hologram Gratings," Bell Syst. Tech. J. 48, 2909-2947 (1969).

7. A. M. Glass, A. M. Johnson, D. H. Olson, W. Simpson, and A. A. Ballman, "Four-wave Mixing in Semi-insulating InP and GaAs Using The Photorefractive Effect," Appl. Phys. Lett. 44, 948-950 (1984). 